\title{
PRODUÇÃO DE ETANOL POR Saccharomyces cerevisiae EM BATELADA UTILIZANDO PROCESSO EXTRATIVO COM DIÓXIDO DE CARBONO
}

\author{
J. L. S. SONEGO ${ }^{1}$, G. Y. RODRIGUEZ ${ }^{1}$, A. J. G. CRUZ $^{1}$ e A. C. BADINO ${ }^{1}$ \\ ${ }^{1}$ Universidade Federal de São Carlos, Programa de Pós-Graduação em Engenharia \\ Química \\ E-mail para contato: silveirasonego@gmail.com
}

\begin{abstract}
Resumo - No presente trabalho foi avaliada a produção de etanol por fermentação extrativa em batelada empregando $\mathrm{CO}_{2}$ como gás de araste. $\mathrm{Na}$ primeira etapa foram analisadas as influências da vazão específica de $\mathrm{CO}_{2}$ e da temperatura no esgotamento do etanol, utilizando como critério de desempenho o fator de arraste $\left(\mathrm{F}_{\mathrm{A}}\right)$, sendo que os resultados mostraram que a vazão específica do gás foi a variável que apresentou uma maior influência no arraste. Numa segunda etapa foi realizada uma fermentação convencional em batelada (sem arraste) em duplicata para a modelagem do processo e obtenção dos parâmetros cinéticos e, na sequência, foram realizadas simulações das fermentações extrativas iniciando a etapa de extração em 3 e $4 \mathrm{~h}$ de cultivo. O emprego da extração de etanol por arraste com $\mathrm{CO}_{2}$ gerou aumentos de produtividade em etanol (em g/L-h) de $25 \%$ e $21,4 \%$, respectivamente, para os arrastes com início em 3 e $4 \mathrm{~h}$ de processo, demonstrando a superioridade da fermentação extrativa com $\mathrm{CO}_{2}$ em relação ao processo convencional.
\end{abstract}

\section{INTRODUÇÃO}

Nos últimos anos, o impacto ambiental associado ao excessivo uso de produtos derivados do petróleo, em especial os combustíveis, tem levado a uma busca crescente por bioenergia. Neste cenário, o etanol por ser renovável e ambientalmente correto, apresenta-se como uma das melhores alternativas como fonte de energia (BAI et al., 2008).

A produção de etanol por via fermentativa é o processo mais utilizado e aplicado pela indústria. Consiste em uma série de reações químicas catalisadas por um microrganismo, sendo geralmente empregada a levedura Saccharomyces cerevisiae. Este microrganismo é utilizado, principalmente, devido à sua maior produtividade, adaptação ao meio e robustez.

O etanol é um dos principais componentes tóxicos para a fermentação alcoólica, agindo como inibidor de sua própria biossíntese. Como resultado desse efeito, tem-se uma queda proporcional nas velocidades de crescimento celular e de produção de etanol, conforme a concentração desse inibidor aumenta no caldo de fermentação (MAIORELLA et al., 1983).

Em decorrência do efeito inibidor, quando a concentração de etanol no caldo atinge valores da ordem de 10 a $12 \% \mathrm{v} / \mathrm{v}$, a produtividade do processo é fortemente afetada, resultando em fermentações longas e incompletas. 
Uma alternativa para contornar o efeito da inibição causada pelo etanol e assim obter uma alta produtividade no processo fermentativo é a utilização de técnicas de extração do etanol do meio fermentativo à medida que ele é produzido (SCHÜGERL, 2000).

Para realizar a extração do etanol combinada com a fermentação pode ser aplicada a operação de esgotamento (stripping) empregando um gás de arraste. Essa técnica corresponde a um processo em que compostos voláteis presentes em uma solução são transferidos para uma fase gasosa, por meio do contato direto da solução com uma corrente gasosa insolúvel. A mesma se apresenta como uma ferramenta promissora para minimizar os efeitos da inibição causada pelo produto, possibilitando um aumento na produtividade do processo fermentativo empregando-se o mesmo volume de trabalho.

Nesse contexto, o objetivo deste trabalho foi avaliar a produção de etanol de primeira geração $(1 \mathrm{G})$ por fermentação extrativa empregando $\mathrm{CO}_{2}$ como gás de araste.

\section{MATERIAL E MÉTODOS}

\subsection{Estudo do Arraste de Etanol - Influência da Vazão Específica de Alimentação de $\mathrm{CO}_{2}$ e da Temperatura de Operação}

Numa primeira etapa, com a finalidade de avaliar quais variáveis apresentam maior influência no processo de esgotamento com dióxido de carbono, foi realizado um estudo do arraste de etanol a partir de uma solução hidroalcóolica, variando a vazão específica de alimentação de $\mathrm{CO}_{2}(\phi)$ e a temperatura da solução $(\mathrm{T})$.

Equipamento: Os experimentos para estudo do arraste foram conduzidos em reator pneumático tipo coluna de bolhas encamisado com volume útil de $5 \mathrm{~L}$ (BADINO et al., 2007). Foi utilizada uma solução hidroalcoólica com concentração de etanol de $10{ }^{\circ} \mathrm{GL}$ e o gás de arraste foi dióxido de carbono industrial armazenado em torpedo. Para controlar a vazão de gás foi empregado um fluxômetro de massa AALBORG, modelo GFC 37. A temperatura do reator foi mantida utilizando banho termostatizado. Durante a realização dos experimentos foi usado um termômetro digital marca Hanna Instruments, modelo HI 147-00 para monitorar a temperatura da solução. Foram retiradas amostras a cada 1 hora durante 6 horas de esgotamento.

Planejamento experimental: Para o estudo das variáveis mais importantes no processo de arraste (stripping) foi realizado um Delineamento Composto Central Rotacional com dois níveis, utilizando o software Statistica 7.0, tendo $2^{2}$ pontos fatoriais 2 x 2 pontos axiais e 3 repetições no ponto central, totalizando 11 experimentos (RODRIGUES E IEMMA, 2009).

Na Tabela 1 são mostrados os valores utilizados no planejamento experimental para as duas variáveis independentes analisadas.

Como variável resposta foi calculado o Fator de Arraste $\left(\mathrm{F}_{\mathrm{A}}\right)$, definido pela Equação 1.

$$
F_{A}(\%)=\frac{C_{E 0} \cdot V_{0}-C_{E F} \cdot V_{F}}{C_{E 0} \cdot V_{0}} .100
$$


onde $\mathrm{C}_{\mathrm{E} 0}$ é a concentração inicial de etanol $\left(\mathrm{g} \cdot \mathrm{L}^{-1}\right), \mathrm{C}_{\mathrm{EF}}$ é a concentração final de etanol $\left(\right.$ g. $\left.\mathrm{L}^{-1}\right), \mathrm{V}_{0}$ é o volume inicial da solução (L) e $\mathrm{V}_{\mathrm{F}}$ é o volume final da solução (L).

Tabela 1 - Valores reais e codificados das duas variáveis independentes

\begin{tabular}{lllllll}
\hline & & \multicolumn{5}{c}{ Nível } \\
\hline Variável & Código & $-1,41$ & -1 & 0 & +1 & $+1,41$ \\
$\phi(\mathbf{v v m})$ & $\mathrm{X} 1$ & 0,38 & 1,00 & 2,50 & 4,00 & 4,62 \\
$\mathbf{T}\left({ }^{\circ} \mathbf{C}\right)$ & $\mathrm{X} 2$ & 29,20 & 30,00 & 32,00 & 34,00 & 34,82 \\
\hline
\end{tabular}

\subsection{Avaliação das Fermentações Alcoólicas Convencional e Extrativa}

Com o objetivo de realizar a modelagem cinética do processo de fermentação alcoólica para subsequente simulação da fermentação extrativa foi realizado inicialmente um ensaio fermentativo em duplicata.

Micro-organismo e Meio de cultura: Saccharomyces cerevisiae (marca Fleischmann) na forma liofilizada com concentração inicial de $15 \mathrm{~g} . \mathrm{L}^{-1}$ foi utilizada neste estudo.

A composição do meio de cultura consistiu de (g. $\left.\mathrm{L}^{-1}\right)$ : sacarose (açúcar cristal) (180), $\mathrm{KH}_{2} \mathrm{PO}_{4}(5,6), \mathrm{MgSO}_{4} 7 \mathrm{H}_{2} \mathrm{O}(1,4)$, extrato de levedura $(6,8)$ e ureia $(5,32) . \mathrm{O}$ pH inicial do caldo de fermentação foi ajustado para 4,6 com adição de acido clorídrico (1 M).

Procedimento experimental: $\mathrm{O}$ reator empregado nas fermentações convencional e extrativa foi o mesmo empregado nos experimentos de arraste, porém esse reator foi adaptado com um sistema de agitação mecânica com o objetivo de garantir a agitação nas horas iniciais do processo, onde ainda não estava sendo alimentado o gás de arraste.

A levedura foi inoculada na forma seca, sendo nesse momento a agitação aumentada para $800 \mathrm{rpm}$ a fim de facilitar a completa dissolução dos flocos de levedura e promover sua mistura no meio. Também, no momento inicial do cultivo foi feito o uso de antiespumante para impedir formação excessiva de espuma. Foi adotado um tempo de aclimatação da levedura de $15 \mathrm{~min}$, antes de ser retirada a primeira amostra. Após o início do processo fermentativo, a agitação foi mantida em $250 \mathrm{rpm}$ até o final da batelada. A temperatura foi mantida em $34{ }^{\circ} \mathrm{C}$ fazendo circular água proveniente de um banho termostatizado pela camisa do biorreator. $\mathrm{O} \mathrm{pH}$ foi acompanhado durante o processo utilizando $\mathrm{pH}$-metro da marca Qualxtro, modelo QX1500. Amostras de $30 \mathrm{~mL}$ foram retiradas a cada $1 \mathrm{~h}$.

Determinações analíticas: A concentração celular foi determinada por análise termogravimétrica da biomassa seca após centrifugação da amostra a $10.000 \mathrm{rpm}$ por $10 \mathrm{~min}$ a $4{ }^{\circ} \mathrm{C}$ e lavagem do precipitado 2 vezes com água destilada. A secagem do precipitado foi feita em estufa à $80^{\circ} \mathrm{C}$ por $24 \mathrm{~h}$. Após este período, realizou-se a pesagem do sedimento seco.

As concentrações de sacarose, glicose e frutose e etanol foram determinadas empregando HPLC (Waters), equipado com detector de índice de refração. Foi utilizada coluna Sugar-Pak I ( 300 × 6,5 mm, $10 \mu \mathrm{m}$, Waters) operada a $80^{\circ} \mathrm{C}$. Água ultrapura foi usada 
como eluente a uma vazão de $0,5 \mathrm{~mL} \cdot \mathrm{min}^{-1}$. Os padrões foram soluções de sacarose, glicose, frutose e etanol com concentrações de 0,1 a 8 g.L $\mathrm{L}^{-1}$.

Modelagem matemática do processo: Para um processo fermentativo operando em batelada e admitindo que a geração do produto seja associada ao crescimento celular, as equações de balanço de massa para células $(\mathrm{Cx})$, substrato $(\mathrm{Cs})$ e produto $(\mathrm{Cp})$ resultam em um sistema de equações diferenciais ordinárias conforme mostrado nas Equações de 2 a 4 :

$$
\begin{aligned}
& \frac{d C x}{d t}=\mu \cdot C x \\
& \frac{d C s}{d t}=-\frac{1}{Y_{X / S}} \cdot \mu \cdot C x \\
& \frac{d C p}{d t}=\frac{Y_{P / S}}{Y_{X / S}} \cdot \mu \cdot C x
\end{aligned}
$$

onde Cx é a concentração celular $\left(\mathrm{g} . \mathrm{L}^{-1}\right), \mu$ é a velocidade específica de crescimento celular $\left(\mathrm{h}^{-1}\right)$, Cs é a concentração de substrato limitante $\left(\mathrm{g} \cdot \mathrm{L}^{-1}\right)$, no caso a soma das concentrações de glicose e frutose, $\mathrm{Cp}$ é a concentração de etanol $\left(\mathrm{g} . \mathrm{L}^{-1}\right), \mathrm{Y}_{\mathrm{X} / \mathrm{S}}$ é o coeficiente de rendimento de substrato a células $\left(\mathrm{g}_{\mathrm{X}} \cdot \mathrm{g}_{\mathrm{S}}{ }^{-1}\right)$ e $\mathrm{Y}_{\mathrm{P} / \mathrm{S}}$ é o coeficiente de rendimento de substrato a etanol $\left(\mathrm{g}_{\mathrm{P}} \cdot \mathrm{g}_{\mathrm{S}}^{-1}\right)$.

Como modelo de crescimento foi utilizado o modelo cinético de Andrews-Levenspiel, considerando as inibições de substrato e produto dado pela Equação 5.

$$
\mu=\mu_{\max } \cdot \frac{C s}{\left(K_{S}+C s+\frac{C s^{2}}{K_{i S}}\right)} \cdot\left(1-\frac{C p}{C p_{\max }}\right)^{n}
$$

onde $\mu_{\max }$ é a velocidade específica máxima de crescimento celular $\left(\mathrm{h}^{-1}\right), \mathrm{K}_{\mathrm{S}}$ é a constante de saturação $\left(\mathrm{g} . \mathrm{L}^{-1}\right), \mathrm{K}_{\mathrm{iS}}$ é a constante de inibição pelo substrato (g.L $\left.\mathrm{L}^{-1}\right), \mathrm{Cp}_{\max }$ é a concentração máxima de etanol a partir da qual cessa o crescimento celular e n é uma constante adimensional.

Os coeficientes de rendimento de substrato a células e a produto (etanol), $\mathrm{Y}_{\mathrm{X} / \mathrm{S}}$ e $\mathrm{Y}_{\mathrm{P} / \mathrm{S}}$, são obtidos pelas Equações 6 e 7.

$$
\begin{gathered}
Y_{X / S}=\frac{C x-C x_{0}}{C s_{0}-C s} \\
Y_{P / S}=\frac{C p-C p_{0}}{C s_{0}-C S}
\end{gathered}
$$

Ajuste do modelo cinético: Para ajuste do modelo aos dados experimentais e estimativa dos parâmetros cinéticos utilizou-se um rotina implementada no software Scilab versão 5.4.1, baseando-se no algoritmo Nelder \& Mead de otimização que fornece um valor mínimo para o resíduo, neste caso definido pela função objetivo apresentada pela Equação (8) (BONOMI e SCHMIDELL, 2001).

$$
R=\sum\left(y_{\exp }-y_{\text {calc }}\right)^{2}
$$


Modelagem da Fermentação Alcoólica com Arraste de Etanol por $\mathrm{CO}_{2}$ (Stripping): Com base nos balanços de massa para a fermentação extrativa, considerando uma vazão de arraste de etanol no biorreator $\left(\dot{m}_{p}\right)$, obtém-se o modelo matemático da fermentação extrativa em batelada, representado pelas Equações 9 a 11.

$$
\begin{aligned}
& \frac{d C x}{d t}=\mu \cdot C x-C x \cdot \frac{1}{V} \cdot \frac{d V}{d t} \\
& \frac{d C s}{d t}=-\frac{1}{Y_{X / S}} \cdot \mu \cdot C x-C S \cdot \frac{1}{V} \cdot \frac{d V}{d t} \\
& \frac{d C p}{d t}=\frac{Y_{P / S}}{Y_{X / S}} \cdot \mu \cdot C x-C p \cdot \frac{1}{V} \cdot \frac{d V}{d t}-\frac{\dot{m}_{p}}{V} \\
& \frac{d V}{d t}=\frac{\dot{m}_{p}+\dot{m}_{A}}{\rho_{A}}
\end{aligned}
$$

onde $\mathrm{V}$ é o volume líquido de caldo (L), $\dot{m}_{p}$ é a vazão mássica de etanol arrastado pela corrente de $\mathrm{CO}_{2}\left(\mathrm{~g} \cdot \mathrm{h}^{-1}\right), \dot{m}_{A}$ é a vazão mássica de água arrastado pela corrente de $\mathrm{CO}_{2}\left(\mathrm{~g} \cdot \mathrm{h}^{-1}\right)$ e $\rho_{\mathrm{A}}$ á a massa específica da água $\left(\mathrm{g} \cdot \mathrm{L}^{-1}\right)$.

A variação no volume do reator durante a fermentação está representada pela Equação 12, considerando a massa específica da solução como sendo igual à da água.

Durante o processo fermentativo ocorre formação de $\mathrm{CO}_{2}$, logo deve-se considerar esta parcela de gás na modelagem. Com base na estequiometria da fermentação alcoólica e fazendo a conversão para unidades de vazão volumétrica, obtém-se a vazão de $\mathrm{CO}_{2}$ gerado naturalmente no processo $\left(\phi_{\mathrm{CO}_{2}} \mathrm{em} \mathrm{L} \cdot \mathrm{min}^{-1}\right)$, dada pela Equação 13.

$$
\phi_{\mathrm{CO}_{2}}=\frac{24,86}{46 \cdot 60} \cdot \frac{Y_{P / S}}{Y_{X / S}} \cdot \mu \cdot C_{x} \cdot V
$$

A integração do sistema de equações diferenciais formado pelas Equações 9, 10, 11 e 12 foi com o algoritmo de Runge-Kutta implementado no software Scilab versão 5.4.1.

\section{RESULTADOS E DISCUSSÃO}

\subsection{Estudo do arraste de etanol - Influência da vazão e da temperatura de operação}

Com os resultados obtidos foi possível verificar as influências da vazão do gás e da temperatura no arraste de etanol (stripping). As contribuições dessas variáveis foram analisadas em função do fator de arraste $\left(\mathrm{F}_{\mathrm{A}}\right)$, conforme mostrado na Tabela 2.

Com base nos resultados obtidos de fator de arraste $\left(\mathrm{F}_{\mathrm{A}}\right)$, pode-se observar que a vazão de gás $(\phi)$ apresenta uma maior influência no arraste na faixa experimental avaliada $(\phi$ e T).

A partir dessas informações foram definidos valores de vazão $(\phi)$ e temperatura $(T)$ a serem empregados nas simulações da fermentação extrativa com arraste de etanol por $\mathrm{CO}_{2}$, 
como 4,0 vvm e $34{ }^{\circ} \mathrm{C}$, respectivamente. Foi adotada a vazão de 4,00 vvm em razão de problemas de instabilidade do fluxômetro de massa na maior vazão, devido provavelmente ao congelamento de $\mathrm{CO}_{2}$ na válvula de expansão.

Tabela 2 - Matriz do planejamento experimental com os valores de Fator de Arraste (FA)

\begin{tabular}{cccccc}
\hline Ensaio & $\mathbf{X}_{\mathbf{1}}$ & $\mathbf{X}_{\mathbf{2}}$ & $\boldsymbol{\phi}(\mathbf{v v m})$ & $\mathbf{T}\left({ }^{\circ} \mathbf{C}\right)$ & $\mathbf{F A}(\boldsymbol{\%})$ \\
\hline $\mathbf{1}$ & $-1,00$ & $-1,00$ & 1,00 & 30,00 & 12,5 \\
$\mathbf{2}$ & $-1,00$ & 1,00 & 1,00 & 34,00 & 8,8 \\
$\mathbf{3}$ & 1,00 & $-1,00$ & 4,00 & 30,00 & 25,7 \\
$\mathbf{4}$ & 1,00 & 1,00 & 4,00 & 34,00 & 21,3 \\
$\mathbf{5}$ & $-1,41$ & 0,00 & 0,38 & 32,00 & 5,9 \\
$\mathbf{6}$ & 1,41 & 0,00 & 4,62 & 32,00 & 30,5 \\
$\mathbf{7}$ & 0,00 & $-1,41$ & 2,50 & 29,20 & 15,3 \\
$\mathbf{8}$ & 0,00 & 1,41 & 2,50 & 34,82 & 20,3 \\
$\mathbf{9}$ & 0,00 & 0,00 & 2,50 & 32,00 & 21,1 \\
$\mathbf{1 0}$ & 0,00 & 0,00 & 2,50 & 32,00 & 20,1 \\
$\mathbf{1 1}$ & 0,00 & 0,00 & 2,50 & 32,00 & 17,9 \\
\hline
\end{tabular}

Com os valores das massas de etanol e água arrastados nos experimentos do planejamento, foi possível obter equações de previsão das vazões de arraste de etanol $\left(\dot{m}_{p}\right)$ e água $\left(\dot{m}_{A}\right)$ do sistema, por meio do ajuste de funções aos dados experimentais, utilizando o software LAB Fit. Essas equações escritas em função da temperatura do sistema (T), da concentração de etanol $\left(\mathrm{C}_{\mathrm{Et}}\right)$ e da vazão específica de $\mathrm{CO}_{2}(\phi)$ são apresentadas na sequência:

$$
\begin{aligned}
& \dot{m}_{p}=0,049 \cdot \emptyset^{0,741} \cdot T^{1,191} \cdot C_{E t}^{0,15} \\
& \dot{m}_{A}=0,513 \cdot \emptyset^{0,595} \cdot T^{0,824}
\end{aligned}
$$

\subsection{Fermentação alcoólica - Modelagem matemática e estimativa de parâmetros}

Os parâmetros $\mathrm{Y}_{\mathrm{X} / \mathrm{S}}$ e $\mathrm{Y}_{\mathrm{P} / \mathrm{S}}$ foram calculados a partir das equações (6) e (7) e os demais foram determinados por ajuste do modelo aos pontos experimentais. Os valores obtidos para os parâmetros foram: $\mathrm{Y}_{\mathrm{X} / \mathrm{S}}=0,046 \mathrm{~g}_{\mathrm{x}} / \mathrm{g}_{\mathrm{S}}, \mathrm{Y}_{\mathrm{P} / \mathrm{S}}=0,46 \mathrm{~g}_{\mathrm{P}} / \mathrm{g}_{\mathrm{S}}, \mu_{\max }=0,50 \mathrm{~h}^{-1}, \mathrm{~K}_{\mathrm{S}}=24,6 \mathrm{~g} . \mathrm{L}^{-1}$, $\mathrm{K}_{\mathrm{iS}}=30,4$ g. $\mathrm{L}^{-1} \mathrm{Cp}_{\max }=85,0$ g. $\mathrm{L}^{-1}$ e $\mathrm{n}=0,89$. A Figura 1 ilustra o excelente ajuste do modelo aos valores experimentais de concentrações de substrato, células e etanol.

Com modelo de inibição mista proposto (Andrews-Levenspiel) foi possível estimar os parâmetros cinéticos da fermentação alcoólica pela levedura e observar que o modelo, descreveu adequadamente o comportamento dinâmico do processo fermentativo convencional. 


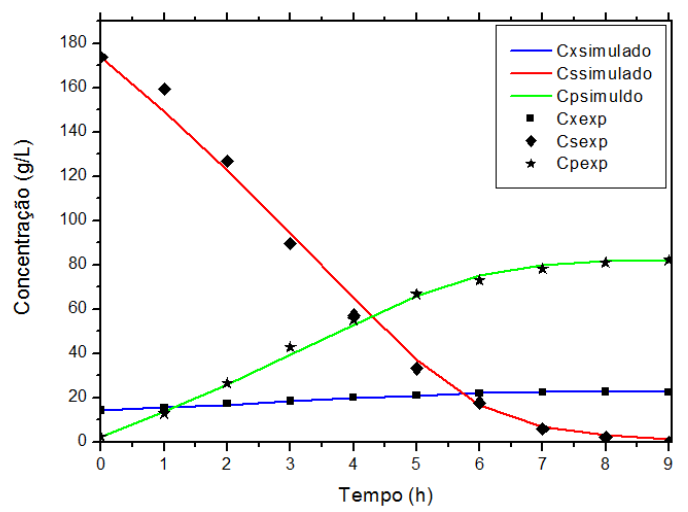

Figura 1 - Variação das concentrações de biomassa, substrato e etanol ao longo da fermentação convencional em batelada.

\subsection{Simulação da fermentação extrativa com arraste de etanol por $\mathrm{CO}_{2}$}

Com as equações do modelo da fermentação com arraste e fazendo uso das equações de previsão das vazões de arraste de etanol e água (Equações 14 e 15) mais os parâmetros cinéticos estimados foram realizadas simulações para avaliar o efeito da extração na dinâmica do bioprocesso com arraste de etanol por $\mathrm{CO}_{2}$. No caso da fermentação extrativa, considerouse o início do arraste na $3^{\mathrm{a}}$ e $4^{\mathrm{a}}$ horas do processo. A Figuras 2 ilustra a simulação do processo fermentativo com arraste (simulado) e sem arraste (experimental).

(a)

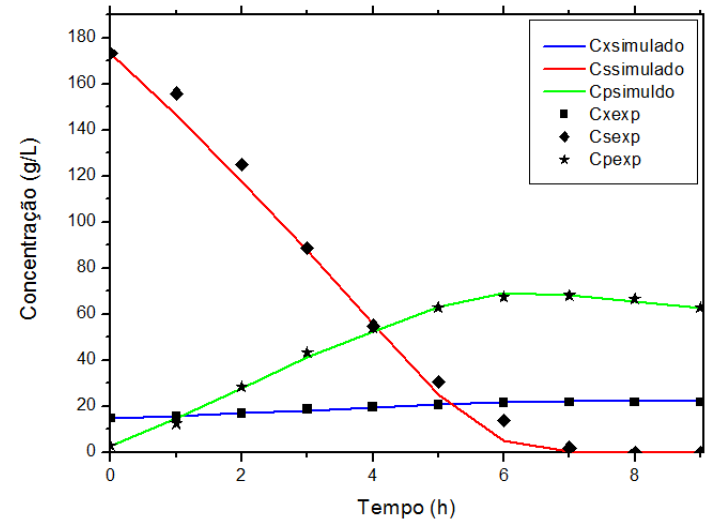

(b)

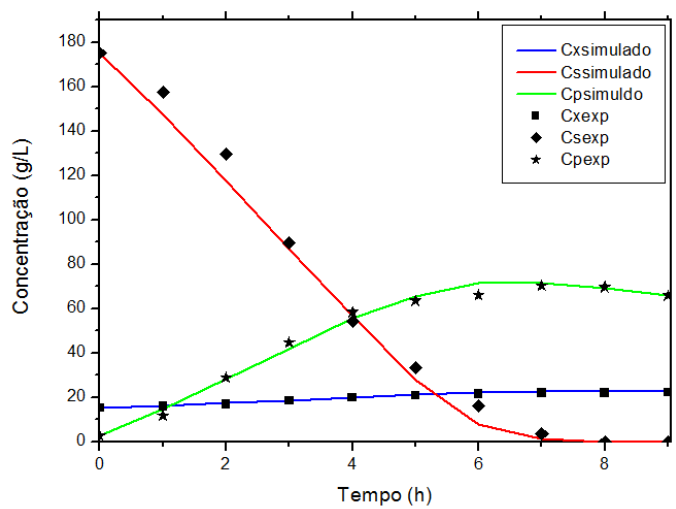

Figura 2 - Valores experimentais e simulados para $(\mathrm{Cx}),(\mathrm{Cs})$ e $(\mathrm{Cp})$ com arraste iniciando na $3^{\mathrm{a}} \mathrm{h}(\mathrm{a})$ e $4^{\mathrm{a}} \mathrm{h}(\mathrm{b})$ de cultivo.

Analisando as Figuras 2 e 3, pode-se observar que o modelo proposto para descrever a fermentação extrativa mostrou-se adequado prevendo o comportamento do processo ao longo do tempo. Pode-se verificar o efeito positivo da remoção do etanol ao longo da fermentação por meio do aumento da velocidade de consumo do substrato, o qual chega a concentrações próximas a zero em torno de sete $\mathrm{h}$ de fermentação, quando o arraste foi iniciado a partir da $3^{\mathrm{a}}$ $\mathrm{h}$ de cultivo. Para o arraste com inicio a partir da $4^{\mathrm{a}} \mathrm{h}$, o substrato terminou em torno de $7,5 \mathrm{~h}$ 
de cultivo. O uso da fermentação extrativa possibilitou ganhos em produtividade de $25 \%$ ( $3^{\mathrm{a}}$ h) e $21,4 \%\left(4^{\mathrm{a}} \mathrm{h}\right)$ para a fermentação extrativa em batelada em relação à convencional sem extração, pois foi possível o consumo de todo substrato em menores intervalos de tempo em comparação com a fermentação convencional (Figura - 1), a qual terminou com $9 \mathrm{~h}$ de cultivo.

\section{CONCLUSÃO}

A vazão de gás apresentou uma forte influência no arraste de etanol durante a operação de stripping nas faixas de temperatura e vazão avaliadas. O modelo híbrido de inibição por substrato e produto (Andrews-Levenspiel) apresentou um bom ajuste aos dados experimentais, podendo ser adotado para simulação das variáveis de interesse em outras condições de cultivo. $\mathrm{O}$ modelo proposto para fermentação extrativa mostrou-se eficaz para previsão do comportamento do processo ao longo do tempo. A fermentação extrativa com $\mathrm{CO}_{2}$ apresentou-se como uma boa alternativa para diminuir o efeito inibidor do etanol sobre a levedura, tornando o processo mais produtivo.

\section{AGRADECIMENTOS}

Os autores agradecem a CAPES e à FAPESP (PITE Proc. 2012/50046-4) pelo auxílio financeiro.

\section{REFERÊNCIAS}

BADINO, A.C.; CERRI, M.O.; HOKKA, C.O. Sistema reacional pneumático e uso do mesmo. Patente licenciada (PI0701608-5), 2007.

BAI, F. W.; ANDERSON, W. A.; MOO-YOUNG, M. Ethanol fermentation Technologies fromsugar and starch feedstocks. Biotechnology Advances v. 26, p. 89-105, 2008.

BONOMI, A. e SCHMIDELL, W. Modelagem matemática e simulação de processos fermentativos. In. BORZANI, W. Biotecnologia Industrial. Vol. 2. Editora Edgard Blucher. São Paulo, 2001. p.123-178.

CHRISTEN, P.; MINER, M.; RENON, H. Ethanol extraction by supported liquid membrane during fermentation. Biotechnology and bioengineering, v. 36, p. 116-123, 1990.

MAIORELLA, B.; BLANCH, H. W.; WILKE, C. R. By-product inhibition effects on ethanolic fermentation by Saccharomyces cerevisiae. Biotechnology and Bioengineering, $\mathrm{v}$. 25, n. 1, p. 103-121, 1983.

RODRIGUES, M.I., IEMMA, A.F. (2005) Planejamento de Experimentos e Otimização de Processos: Uma estratégia Sequencial de planejamentos. Casa do Pão Editora, 2005.

SCHÜGERL, K. Integrated processing of biotechnology products. Biotechnology Advances, 18, 581-599, 2000. 